cytoplasmatic and the viral genome is not inserted into the cell DNA. Cancer development is thus believed to depend on interaction of viral proteins with the cell cycle and/or to result from continuous liver cell damage and regeneration (cell turnover). HCV core, NS3A and NS5A proteins have been shown to inhibit $\mathrm{p} 21^{\text {waf }}$ expression and p53 activity. In addition, HCV core protein inhibits cell apoptosis induced by TNF-alpha and Fas and NS5A transactivates cell cyclins and growth factors.

HIV-HCV coinfection induces significant changes in the natural history of HCV liver disease, rendering HCV persistence more likely, with higher levels of HCV viremia and faster progression of liver disease. This may be due to less effective anti-HCV CD${ }_{4}^{+}$and $\mathrm{CD}_{8}^{+}$cell immune response, as well as to impaired dendritic cell function.

French studies have recently pointed out for a significant increase in HCV-related morbidity and mortality among people living with HIV/AIDS under HAART. Better knowledge about this infection and its relationship with cancer development is essential for the establishment of effective primary and secondary prophylaxis.

\title{
The Descriptive and Molecular Epidemiology of HHV-8 among Population Groups of the Amazon Region of Brazil
}

\author{
R. Ishak, R.N. Martins, L.F.A. Machado, A.C.R. Vallinoto, L. Lobato, T. Sata, M.O.G. Ishak
}

The present study aimed to describe the epidemiology of $H H V-8$ among population groups of the Amazon region of Brazil. Four Indian tribes (Kararao, Arara Laranjal, Tiriyo and Zo'e) and a group of HIV-1 infected and/or with AIDS from the urban population of Belém, Para, were tested for the presence of the virus, using serologic (enzyme immuno assay, ELISA, measuring antibodies to ORF59, early and late protein, lytic cycle, ORF65, late protein of the capsid, lytic cycle, K8.1A and K8.1B, variant forms of the envelope gp, lytic cycle and ORF73, latency maintenance protein) and molecular (gene amplification of the ORF26 and the variable region of VR1, gene K1 segments). The presence of antibodies to $H H V-8$ was detected in 66 samples of the 221 tested of the Indian groups, namely, six (25\%) in the Kararao, 18 (19.6\%) in the Arara Laranjal, 24 (42.9\%) in the Tiriyo and 18 (36.7\%) in the Zo'e. Out of the 477 HIV-1 group, 74 (15.5\%) were seroreactive to HHV-8. The ORF26 region was amplified in seven samples, one of the Arara Laranjal, one of the Tiriyo, two of the Zo'e and three of the HIV-1 infected group. Subtyping procedures showed the presence of subtypes C (Zo'e), E (Tiriyo) and B (HIV-1 infected). Serologic results confirm the high prevalence of $H H V-8$ and the presence of three subtypes in the Amazon region of Brazil. It also describes, for the first time, the prevalence of $H H V-8$ among HIV-1 infected and/or AIDS patients.

\section{New Therapeutic Approaches for HIV and EBV Related Lymphomas}

William J. Harrington, Jr.

Worldwide, more than 40 million individuals are infected with Human Immunodeficiency Virus (HIV). In impoverished countries the number of deaths due to AIDS has rapidly increased, however the infectious and malignant complications of HIV have fallen where potent antiretrovirals (ARV) are widely available. Nonetheless, the prolonged survival of many HIV carriers is likely to result in greater numbers of malignancies among these individuals. Nearly half of all cases of non-Hodgkin's lymphoma (NHL) in AIDS patients are associated with the presence of a gamma herpes virus, Epstein Barr Virus (EBV) or Human Herpes Virus Type 8 (HHV-8).

AIDS NHLs may be categorized into several subtypes. Large cell immunoblastic lymphoma(IBL) and diffuse large cell lymphoma (DLCL) generally occur in the setting of moderate to severe immunosuppression $\left(\mathrm{CD}_{4}^{+}\right.$ 
lymphocyte counts below $100 \mathrm{~mm}^{3} / \mathrm{mL}$ ). IBLs and to a lesser degree DLCLs are often associated with EBV and often express the EBV encoded oncoprotein latent membrane protein-1 (LMP-1). LMP-1 may function in a similar manner to tumor necrosis factor receptors by activating cellular proliferation as well as anti-apoptotic functions (Bcl-2). DLCLs are frequently found to contain genetic alterations in Bcl-6. The consequences of these alterations have not been fully defined.

AIDS related Burkitt's lymphoma (BL) generally occurs in relatively immunocompetent patients. AIDS BLs share features with endemic African BL in that both overexpress the oncogene c-myc due to reciprocal translocations that bring the gene under promotor sequences within the Ig loci. Inactivating mutations and deletions of p53 are also common among all types of BL. A distinguishing feature between AIDS BL and endemic BL is that the former is associated with EBV far less frequently than the latter.

A rapidly fatal subtype of AIDS NHL is Primary Central Nervous System Lymphoma (PCNSL). These tumors occur in the most immunosuppressed patients and are virtually always associated with EBV. Detection of EBV sequences in the CSF by polymerase chain reaction (PCR) coupled with positive Thallium spectroscopy has proven to be an accurate diagnostic tool. Standard therapy with conventional chemotherapy combined with radiation therapy results in only about a 2-month survival.

A peculiar subtype of AIDS NHL is primary effusion lymphoma (PEL). This tumor is one of three proven to be associated with HHV-8; the other two are Kaposi's sarcoma and multicentric Castleman's diseases. PEL usually presents as a malignant pleural, pericardial, or peritoneal effusion and is often initially misdiagnosed as an infectious process. Usually PEL cells contain multiple copies of HHV-8 (60 to 80) as well as EBV. These tumors tend to be rapidly fatal although recent data from our laboratory suggests that some PEL lines are quite sensitive to antiviral agents.

Therapy of AIDS NHL remains disappointing. Polychemotherapy regimens have produced similar results although regimens that combine potent antiretrovirals with conventional chemotherapy may prove superior. In general, our experience has been that patients concomitantly diagnosed with HIV infection and lymphomas do better with antiretroviral and antilymphoma therapy than do those who develop lymphoma after becoming refractory to antiretrovirals. Probably the best reported results for chemotherapy in AIDS NHL were from Dr. Little's group at the National Cancer Institute. Using the EPOCH regimen, the group achieved remission in 22 of 24 patients with a progression free survival of 23 months. These patients had favorable prognostic factors (median $\mathrm{CD}_{4}^{+}$ lymphocyte count of $233 \mathrm{~mm}^{3} / \mathrm{mL}$ ). It is important to comment on the potential enhanced toxicity of adding rituximab to $\mathrm{CHOP}$ chemotherapy that has recently been reported in a large multi-center trial conducted by the AIDS Malignancy Consortium. In this study (AMC 010), the addition of rituximab to standard-dose CHOP led to increased infectious complications and deaths attributable to sepsis. It is possible that delayed recovery of humoral immunity could contribute to this increased risk of life-threatening bacterial infections in HIV-infected patients (manuscript submitted).

\section{Adult T-Cell Leukemia/Lymphoma (ATL) in Bahia, Brazil Achiléa L. Bittencourt}

Adult T-cell leukemia/lymphoma (ATL), an aggressive type of leukemia/lymphoma was first described by Uchiyama et al (1977) in Japan. Subsequent studies demonstrated its association with the human T-cell lymphotropic virus (HTLV-I). One-third of all cases of T-cell lymphoma in Salvador, Bahia, Brazil are found to be associated with this virus, which is endemic in the region.

In the 74 cases of ATL observed in Bahia, the mean age of patients was 48.2 years, lower than that found in patients in Japan, and there were two cases in adolescents of 15 and 17 years of age and one case beginning in 